\title{
Protocolo DE EXTRACCIONES EN ORTODONCIA
}

\author{
Mauricio Villada Castro ${ }^{*}$, Res. Odon. ${ }_{1}$, Andrea Pedroza Garcés, Odon. Ortd. ${ }_{2}$ \\ Universidad Cooperativa de Colombia, sede Envigado, Colombia \\ ${ }_{2}$ Universidad CES, Universidad Cooperativa de Colombia, sede Envigado, Colombia
}

Recibido: 7 de noviembre del 2012 Aprobado: 8 de febrero del 2013

* Autor de contacto: Mauricio Villada Castro, Facultad de Odontología, Universidad Cooperativa de Colombia, 2706466, Carrera 47 n. ${ }^{\circ} 37$ sur 18 , Medellín, Colombia, correo electrónico: mavic6@hotmail.com

Cómo citar este artículo: Villada Castro M, Pedroza Garcés A. Protocolo de extracciones en ortodoncia. Rev. Nac. Odontol. 2013 diciembre; 9 (edición especial): $17-23$.

Resumen. En muchas ocasiones, la extracción dental como resultado de una necesidad de espacio para el tratamiento ortodóncico, crea alteraciones en la cresta alveolar que pueden resultar en problemas funcionales y estéticos para la correcta finalización del tratamiento, por la insuficiente cantidad de tejido óseo. Este problema le exige al especialista hacer un razonamiento ordenado de cada situación clínica individual y considerar una serie de factores propios del paciente, del tratamiento y del profesional al que remiten, que le indiquen el momento apropiado para realizar las exodoncias de su paciente. El propósito de este artículo es hacer una descripción de los cambios del alvéolo dentario posextracción, y sugerir un protocolo que sea apropiado para definir el momento y la mejor manera en que se deban realizar las extracciones dentales de un paciente ortodóncico.

Palabras clave: extracción dental, hueso alveolar, remisión, tratamiento ortodóncico.

\section{PROTOCOL FOR EXTRACTIONS IN ORTHODONTIA}

Abstract. Tooth extraction due to the need for space for orthodontic treatment in many cases causes changes in the alveolar ridge, which can result in functional and aesthetic problems for the successful completion of treatment stemming from an insufficient amount of bone tissue. This problem requires the specialist to analyze each individual clinical situation and consider a number of factors specific to the patient, the treatment and the professional to whom they are referred, who will then determine the appropriate time to perform extractions. The purpose of this article is to describe post-extraction changes in the tooth socket and suggest a protocol that is appropriate for defining when and how best to carry out tooth extractions in orthodontic patients.

Keywords: tooth extraction, alveolar bone, referral, orthodontic treatment. 


\section{Introducción}

En los tratamientos de ortodoncia, la extracción es una decisión meditada y estudiada en la que los ortodoncistas deben analizar qué dientes se van a extraer, cómo se va a distribuir el espacio y qué biomecánica se va a emplear, decisión que se basa en un diagnóstico adecuado. Sin embargo, algunas veces ellos no son conscientes de las consecuencias que una simple extracción dental puede tener sobre los resultados de los tratamientos de ortodoncia $[1,2]$, los cuales pueden ir desde demora en dichos resultados, hasta el no cierre de los espacios o incluso la reapertura de estos después de haber finalizado la terapia.

La mayoría de estas complicaciones obedecen a que el ortodoncista no tiene en cuenta los tiempos de colapso de los rebordes alveolares al planear y remitir sus extracciones, encontrándose que los cambios atróficos progresivos del reborde preceden al cierre de espacios [3].

Con este artículo se pretende:

- Que el ortodoncista conozca los cambios del alvéolo dentario posextracción.

- Sugerir un protocolo de extracción en ortodoncia que tenga en cuenta el momento del tratamiento en que se deben realizar las extracciones, y la manera en que se deben remitir.

\section{Cambios alveolares posextracción}

Se ha demostrado que las dimensiones alveolares se reducen significativamente después de la extracción dental, mostrando mayor pérdida en el ancho y en la altura en el maxilar que en la mandíbula $[4,5]$.

Van der Weijden $[1,6]$ hizo una revisión de literatura para evaluar la cantidad de cambios en el ancho y alto de los rebordes alveolares posextracción, y encontró que después de una extracción dentaria se deben esperar cambios horizontales y verticales tanto en el tejido duro como en el blando, los cuales se producen de manera acelerada entre los primeros tres a seis meses y continúan en promedio hasta los doce meses posextracción, llegando el reborde a perder hasta un $40 \%$ de su altura y un $60 \%$ de amplitud $[2,7,8]$.

Sobre modelos caninos, Araujo ha demostrado un marcado cambio dimensional en el hueso alveolar en los primeros 2 a 3 meses posextracción, siendo más pronunciado el cambio en la región bucal [9]. Este cambio se ha reportado en una magnitud de hasta del
$56 \%$, mientras que la reabsorción ósea en la pared lingual es del 30\% [10].

A nivel histológico, Cardaropoli y Cardaropoli reportan que después de la extracción dentaria ocurre una primera etapa de reabsorción ósea entre la cuarta y quinta semana, estabilizándose después de los seis meses con un rango de 3 a $5 \mathrm{~mm}$ [11]. Asimismo, Schropp et al. [5] encontraron que la reducción bucolingual del alvéolo fue aproximadamente del 50\%, pasando de 12 $\mathrm{mm}$ a $5,9 \mathrm{~mm}$ en los 12 meses siguientes, ocurriendo dos tercios de este cambio en los primeros tres meses después de la extracción.

En su revisión, Chen y Hämmerle [12] reportan que después de 6 meses de haber realizado una extracción dentaria se produce una reabsorción horizontal aproximadamente de $5 \mathrm{~mm}$, que equivale a dos tercios del ancho inicial y una reducción de $4 \mathrm{~mm}$ a nivel vertical.

En conclusión, al extraer un diente se inicia muy rápidamente en el alvéolo dentario una serie de procesos progresivos e irreversibles que incluyen cambios verticales y horizontales, en mayor medida en el aspecto bucal del maxilar y de la mandíbula, causados por la reabsorción y el remodelado óseo [1, 13-15].

\section{Protocolo de extracción en ortodoncia}

Un protocolo de extracción es ideal para buscar que los movimientos dentales hacia los espacios de las extracciones sean previos a los cambios atróficos del reborde alveolar, lo cual exige que el profesional decida cuál es el momento del tratamiento en el que es más apropiado realizar las exodoncias, y elabore un formato de remisión en el que le señale al odontólogo los diferentes factores que debe tener en cuenta para disminuir el riesgo de colapso.

\section{Momento de realizar las extracciones}

Generalmente se ha aceptado que las extracciones con fines ortodóncicos se deben realizar al iniciar el tratamiento en casos de maloclusiones, sin tener en cuenta que algunas veces pasará un lapso importante de tiempo entre el momento en que estas se realicen y el momento en que el clínico usará el espacio dejado por ellas. No tener en cuenta este tiempo ha hecho que - cuando llega a la fase de cierre de espacios- el ortodoncista se encuentre frente a rebordes colapsados que retrasan o impiden el cierre de espacio, y pueden llegar incluso a comprometer los resultados ortodóncicos [16-18]. Estas complicaciones se pueden evitar si las extracciones 
se realizan en la fase del tratamiento ortodóncico que busque utilizar inmediatamente el espacio.

El momento de realizar las extracciones no debe ser el mismo para todos los pacientes y su elección debe ser el resultado de un análisis juicioso de dos puntos:

- La situación clínica o tipo de maloclusión y la secuencia de tratamiento.

- Los dientes que se van a extraer.

\section{Situación clínica y secuencia de tratamiento}

Las situaciones clínicas en las que el ortodoncista -después de hacer un estudio del caso- encuentra que debe hacer extracciones con el fin de lograr resultados adecuados, se pueden agrupar en tres categorías: apiñamiento, coordinación de arcos y manejo de biprotrusiones dentoalveolares. A continuación se mencionará cada una de esas situaciones y se relacionarán con el momento en que se deben hacer las extracciones.

\section{- Apiñamiento severo}

En estos casos la falta de espacio se evidencia en malposición dentaria tipo versiones y gresiones.

En este tipo de situación clínica, al realizar las extracciones, el espacio dejado por ellas va a ser usado inmediatamente para la alineación dental. Por tanto, en las situaciones clínicas en las que el espacio se use en la fase de alineación y nivelación, las extracciones se deben realizar al inicio del tratamiento, cuando el riesgo de colapso de reborde es mínimo.

\section{- Coordinación de arcos}

En esta situación clínica se incluyen las desviaciones de línea media, los casos de maloclusión clase II $[19,20]$ y clase III [21] de origen dentoalveolar o esquelético en las cuales el paciente quiere camuflar el problema y la extracción dental ayuda a lograr una adecuada relación de los arcos.

En situaciones en las que no hay malposiciones individuales, el ortodoncista usará el espacio de las extracciones en una fase intermedia del tratamiento. Por tanto, las extracciones se deben programar después de haber alineado y nivelado los arcos, en el momento en que se esté en un arco de trabajo que permita iniciar el cierre biomecánico de los espacios. Estos casos se consideran de riesgo moderado para colapso, ya que los estudios han mostrado cambios dimensionales posextracción en el arco superior e inferior $[4,11]$.

\section{- Manejo de biprotusión dentoalveolar}

En estos casos, el ortodoncista usará el espacio de la extracciones en una fase intermedia del tratamiento, $y$ por tanto estas se deben programar cuando se esté en un arco de trabajo en el que se pueda iniciar el cierre biomecánico de los espacios inmediatamente después de las extracciones [22].

Estos casos se consideran de riesgo moderado para colapso en el arco superior e inferior, por los diferentes patrones de remodelado que se mencionaron anteriormente.

\section{Dientes que se van a extraer}

Al igual que el punto anterior, la influencia que tiene el diente a extraer [23-25] sobre el momento de la extracción se explica en el lapso que transcurrirá entre el momento de hacer la extracción y el cierre biomecánico del espacio, aceptándose que entre más posterior sea la extracción y más anterior el problema (apiñamientos o biprotrusiones), mayor será el riesgo de colapso, ya que pasará más tiempo entre la extracción y el cierre completo del espacio [26-29].

\section{Formato de remisión de extracciones con fines ortodóncicos}

En muchos casos, las extracciones en ortodoncia se remiten de manera rápida e inconsciente, cometiendo errores tanto en forma como en contenido [30]. Los errores de forma se reflejan en la falta de formatos específicos, usando, en algunas ocasiones, hojas de prescripción de medicamentos, las cuales no son adecuadas para tal fin.

Los errores de contenido se pueden evidenciar en la incapacidad del odontólogo que recibe la remisión para resolver preguntas como ¿cuál es la razón de la extracción?, ¿el paciente es de alto o bajo riesgo de colapso?, ¿qué precauciones se deben tener en relación con la técnica y el manejo posoperatorio? [31,32].

A continuación se darán los lineamientos en cuanto a la información que debe tener una remisión para extracciones en ortodoncia.

\section{Identificación}

En este campo se hace especial énfasis en la edad y el sexo del paciente. Se ha encontrado que los pacientes adultos presentan una membrana periodontal menos vascularizada y estrecha, así como un tejido óseo más avascular, denso y calcificado [33], haciendo que los movimientos dentales sean a una tasa mucho menor; por tanto, en caso de remitir para la realización de extracciones, es importante que durante el procedimiento 
se controlen todas la variables relacionadas con la técnica y el posoperatorio, con el fin de disminuir el colapso del reborde.

El sexo tiene gran relevancia clínica en el caso de las extracciones, encontrándose mayor grado de colapso posextracción en mujeres con respecto a los hombres, lo cual se explica en los cambios hormonales y metabólicos [6,34].

\section{Anamnesis}

El segundo componente de un formato de remisión debe contener la anamnesis del paciente y el examen físico. En este punto se debe diferenciar entre el paciente sano y aquel que tiene algún compromiso sistémico [35].

Con respecto al riesgo de colapso, es de especial importancia controlarlo en los pacientes con enfermedades de carácter endocrino y con problemas metabólicos como en la osteoporosis y la obesidad. En el caso de pacientes con osteoporosis, como ellos tienen alterado el metabolismo del calcio, van a presentar tanto una disminución en el movimiento dental, como una alteración en los procesos de cicatrización posextracción. Las personas obesas, por su parte, tienen una regulación no equilibrada entre hormonas y citoquinas que hace que el proceso de reabsorción y aposición ósea no se dé en iguales proporciones [36].

Por tanto, es de gran importancia controlar todas las variables relacionadas con la técnica de extracción y con el manejo posextracción, ya que es necesario disminuir el riesgo de colapso en estos pacientes que inician con una condición comprometida.

\section{Motivo de la remisión}

En el motivo se debe explicar detalladamente el procedimiento que se requiere, siendo explícito no sólo en cuáles son los dientes a extraer sino en la técnica que dé mejores condiciones cicatrizales y que conlleve a la prevención o disminución de la pérdida del reborde alveolar.

Algunas de las técnicas de extracción que podemos solicitar son la extracción simple, la atraumática, con odontosección y la extracción con preservación de reborde, las cuales describiremos a continuación.

\section{- Extracción simple}

Este tipo de procedimiento se indica en pacientes con riesgo mínimo de colapso, y son:
- Maloclusiones con grandes versiones y gresiones, o en las que los dientes adyacentes se van a mover muy rápidamente a los sitios de extracción.

- Cuando los dientes a extraer tengan adecuadas paredes vestibulares y linguales.

- Pacientes que por su edad, sexo y condiciones sistémicas tengan riesgo mínimo de colapso.

Siempre que se soliciten extracciones simples se debe especificar que no haya colapso de tablas óseas y que la sutura sea sólo de aproximación para preservar el coágulo.

Finalmente, aunque a legislación no sea manifiesta al respecto, es ideal especificar los dientes a extraer no sólo apoyándose en la nomenclatura internacional, sino también en el nombre y la ubicación que tiene dicho diente.

\section{- Extracción atraumática}

Los métodos atraumáticos de extracción tienen como objetivo preservar la cresta alveolar y su técnica consiste en usar sólo el elevador para realizar la exodoncia, ya que este realiza menos fuerza haciendo el procedimiento más predecible.

En las extracciones para ortodoncia esta técnica se indica en las siguientes situaciones:

- Extracción de anteroinferiores, ya que tienen tablas óseas vestibulares y linguales muy delgadas que se pueden fracturar fácilmente.

- Cuando el diente a extraer tiene gran prominencia radicular, lo cual está relacionado con una tabla ósea vestibular frágil y delgada [37].

\section{- Extracción con odontosección}

En ortodoncia este tipo de técnicas se usa en algunas maloclusiones que por sus características requieren la exodoncia de molares inferiores y que se hace con el fin de evitar el colapso del reborde, prefiriéndose extraer primero el segmento mesial evitando así el colapso de la porción distal.

\section{- Extracción con preservación de reborde}

Los conceptos de "preservación del alvéolo posextracción" y de "terapia de extracción avanzada" usan materiales de relleno (hueso autógeno, aloinjertos, injertos aloplásticos y xenoinjertos) con el fin de mantener la estabilidad dimensional del hueso alveolar posextracción [2, 38-41]. 
Este tipo de procedimientos está indicado en casos en los que vaya a pasar un gran lapso de tiempo entre el momento de realizar la extracción y el momento del cierre del espacio, disminuyendo con estos procedimientos el riesgo de colapso del reborde [4, 42-46].

\section{Precauciones}

De acuerdo con Bragger y colaboradores, para los cuidados posoperatorios se debe recomendar al paciente el uso durante un mes de enjuagues bucales a base de clorhexidina al $0,12 \%$, ya que se ha encontrado que esto mantiene el nivel óseo de la cresta alveolar posextracción $[47,48]$. Igualmente es importante recomendarle al paciente que no fume, ya que el cigarrillo, según Saldanha y colaboradores [49], puede afectar la altura del reborde alveolar

De acuerdo con Araujo y Lindhe, usar colgajos o no hacerlo para realizar la exodoncia puede alterar el proceso cicatrizal del alvéolo. En el 2009, estos autores encontraron diferencias entre los dos grupos tratados, aunque concluyeron que la alteración dimensional por levantar un colgajo durante la extracción es a corto plazo [50].

\section{Conclusiones}

- En muchas ocasiones se realizan extracciones dentales remitidas por el ortodoncista, sin prever qué cambios va a sufrir el alvéolo posextracción y sin tener en cuenta que estos cambios pueden alterar la biomecánica del tratamiento.

- Factores como el tipo de maloclusión y el diente a extraer hacen que las extracciones dentales del paciente ortodóncico no se deban realizar en la misma fase del tratamiento, con el fin de evitar el colapso de los rebordes óseos alveolares.

- El ortodoncista debe manejar un formato adecuado de remisión para la realización de extracciones dentales en sus pacientes. Esto permite una adecuada interconsulta con el otro profesional y minimiza los errores por falta de información.

\section{Referencias}

[1] Tan WL, Wong TL, Wong MC, Lang NP. A systematic review of post-extractional alveolar hard and soft tissue dimensional changes in humans. Clin Oral Implants Res. 2012; 23(5): 1-21.
[2] Ashman A. Ridge preservation: important buzzwords in dentistry. Gen Dent. 2000; 48(3): 304-12.

[3] Mirmarashi B, Torbati A, Aalam A, Chee W. Orthodontically assisted vertical augmentation in the esthetic zone. J Prosthodont. 2010; 19(3): 235-9.

[4] Iasella JM, Greenwell H, Miller RL, Hill M, Drisko C, Bohra AA et al. Ridge preservation with freeze-dried bone allograft and a collagen membrane compared to extraction alone for implant site development: a clinical and histologic study in humans. J Periodontol. 2003; 74(7): 990-9.

[5] Schropp L, Wenzel A, Kostopoulos L, Karring T. Bone healing and soft tissue contour changes following singletooth extraction: a clinical and radiographic 12-month prospective study. Int J Periodontics Restorative Dent. 2003; 23(4): 313-23.

[6] Van der Weijden F, Dell'Acqua F, Slot DE. Alveolar bone dimensional changes of post-extraction sockets in humans: a systematic review. J Clin Periodontol. 2009; 36(12): 1048-58.

[7] Bartee BK. Extraction site reconstruction for alveolar ridge preservation. Part 1: rationale and materials selection. J Oral Implantol. 2001; 27(4): 187-93.

[8] Molina JN. Preservación del reborde alveolar. Por qué y cuándo. Periodoncia y Oseointegración. 2007; 17(4):9.

[9] Araujo MG, Lindhe J. Dimensional ridge alterations following tooth extraction. An experimental study in the dog. J Clin Periodontol. 2005; 32(2): 212-8.

[10] Botticelli D, Berglundh T, Lindhe J. Hard-tissue alterations following immediate implant placement in extraction sites. J Clin Periodontol. 2004; 31(10): 820-8.

[11] Cardaropoli D, Cardaropoli G. Preservation of the postextraction alveolar ridge: a clinical and histologic study. Int J Periodontics Restorative Dent. 2008; 28(5): 469-77.

[12] Chen ST, Wilson TG, Jr., Hammerle CH. Immediate or early placement of implants following tooth extraction: review of biologic basis, clinical procedures, and outcomes. Int J Oral Maxillofac Implants. 2004; 19(Suppl): 12-25.

[13] Proff P, Bayerlein T, Fanghanel J, Gerike W, Bienengraber V, Gedrange T. The application of bone graft substitutes for alveolar ridge preservation after orthodontic extractions and for augmentation of residual cleft defects. Folia Morphol (Warsz). 2006; 65(1): 81-3.

[14] Oghli AA, Steveling H. Ridge preservation following tooth extraction: a comparison between atraumatic extraction and socket seal surgery. Quintessence Int. 2010; 41(7): 605-9.

[15] Amler MH. The time sequence of tissue regeneration in human extraction wounds. Oral Surg Oral Med Oral Pathol. 1969; 27(3): 309-18. 
[16] Ribarevski R, Vig P, Vig KD, Weyant R, O’Brien K. Consistency of orthodontic extraction decisions. Eur J Orthod. 1996; 18(1): 77-80.

[17] Chiqueto K, Janson G, De Almeida CT, Storniolo JM, Barros SE, Henriques JF. Influence of root parallelism on the stability of extraction-site closures. Am J Orthod Dentofacial Orthop. 1999; 139(6): e505-10.

[18] Reichert C, Wenghofer M, Gotz W, Jager A. Pilot study on orthodontic space closure after guided bone regeneration. J Orofac Orthop. 2011; 72(1): 45-50.

[19] Scott Conley R, Jernigan C. Soft tissue changes after upper premolar extraction in Class II camouflage therapy. Angle Orthod. 2006; 76(1): 59-65.

[20] Luppanapornlarp S, Johnston LE, Jr. The effects of premolar-extraction: a long-term comparison of outcomes in "clear-cut" extraction and nonextraction Class II patients. Angle Orthod. 1993; 63(4): 257-72.

[21] Rabie AB, Wong RW, Min GU. Treatment in Borderline Class III Malocclusion: Orthodontic Camouflage (Extraction) Versus Orthognathic Surgery. Open Dent J. 2008; 2: 38-48.

[22] Leonardi R, Annunziata A, Licciardello V, Barbato E. Soft tissue changes following the extraction of premolars in nongrowing patients with bimaxillary protrusion. A systematic review. Angle Orthod. 2010; 80(1): 211-6.

[23] Peck S, Peck H. Frequency of tooth extraction in orthodontic treatment. Am J Orthod. 1979; 76(5): 491-6.

[24] Bernstein L. Edward H. Angle versus Calvin S. Case: extraction versus nonextraction. Part I. Historical revisionism. Am J Orthod Dentofacial Orthop. 1992; 102(5): 464-70.

[25] Seddon JL. Extraction of four first molars: a case for a general practitioner? J Orthod. 2004; 31(2): 80-5.

[26] Zhylich D, Suri S. Mandibular incisor extraction: a systematic review of an uncommon extraction choice in orthodontic treatment. J Orthod. 2011;38(3): 185-95; quiz 231.

[27] Weintraub JA, Vig PS, Brown C, Kowalski CJ. The prevalence of orthodontic extractions. Am J Orthod Dentofacial Orthop. 1989; 96(6): 462-6.

[28] Sandler PJ, Atkinson R, Murray AM. For four sixes. Am J Orthod Dentofacial Orthop. 2000; 117(4): 418-34.

[29] Canut JA. Ortodoncia clínica y terapéutica. 2a . ed. Barcelona: Masson S.A.; 2000.

[30] Ludke RL. An examination of the factors that influence patient referral decisions. Med Care. 1982; 20(8): 782-96.

[31] Bustamante R. Remisión de pacientes en una unidad regional de salud, Colombia. Bol Of Sanit Panam. 1985; 99(1): 15.
[32] De Bondt B, Aartman IH, Zentner A. Referral patterns of Dutch general dental practitioners to orthodontic specialists. Eur J Orthod. 2010; 32(5): 548-54.

[33] Nowzari H, Yorita FK, Chang HC. Periodontally accelerated osteogenic orthodontics combined with autogenous bone grafting. Compend Contin Educ Dent. 2008; 29(4): 200-6; quiz 7, 18.

[34] Tyrovola JB, Spyropoulos MN. Effects of drugs and systemic factors on orthodontic treatment. Quintessence Int. 2001; 32(5): 365-71.

[35] Patel A, Burden DJ, Sandler J. Medical disorders and orthodontics. J Orthod. 2009; 36(Suppl): 1-21.

[36] Neeley WW, 2nd, Gonzales DA. Obesity in adolescence: implications in orthodontic treatment. Am J Orthod Dentofacial Orthop. 2007; 131(5): 581-8.

[37] Nevins M, Camelo M, De Paoli S, Friedland B, Schenk RK, Parma-Benfenati $S$ et al. A study of the fate of the buccal wall of extraction sockets of teeth with prominent roots. Int J Periodontics Restorative Dent. 2006; 26(1): 19-29.

[38] Tiefengraber J, Diedrich P, Fritz U, Lantos P. Orthodontic space closure in combination with membrane supported healing of extraction sockets (MHE) a pilot study. J Orofac Orthop. 2002; 63(5): 422-8.

[39] Hsiong SX, Mooney DJ. Regeneration of vascularized bone. Periodontol 2000. 2006; 41: 109-22.

[40] Araujo MG, Carmagnola D, Berglundh T, Thilander B, Lindhe J. Orthodontic movement in bone defects augmented with Bio-Oss. An experimental study in dogs. J Clin Periodontol. 2001; 28(1): 73-80.

[41] Wang HL, Kiyonobu K, Neiva RF. Socket augmentation: rationale and technique. Implant Dent. 2004; 13(4): 286-96.

[42] McAllister BS, Haghighat K. Bone augmentation techniques. J Periodontol. 2007; 78(3): 377-96.

[43] Hossain MZ, Kyomen S, Tanne K. Biologic responses of autogenous bone and beta-tricalcium phosphate ceramics transplanted into bone defects to orthodontic forces. Cleft Palate Craniofac J. 1996; 33(4): 277-83.

[44] Cardaropoli D, Re S, Manuzzi W, Gaveglio L, Cardaropoli G. Bio-Oss collagen and orthodontic movement for the treatment of infrabony defects in the esthetic zone. Int J Periodontics Restorative Dent. 2006; 26(6): 553-9.

[45] Re S, Corrente G, Abundo R, Cardaropoli D. Orthodontic movement into bone defects augmented with bovine bone mineral and fibrin sealer: a reentry case report. Int J Periodontics Restorative Dent. 2002; 22(2): 138-45. 
[46] Simon BI, Von Hagen S, Deasy MJ, Faldu M, Resnansky D. Changes in alveolar bone height and width following ridge augmentation using bone graft and membranes. J Periodontol. 2000; 71(11): 1774-91.

[47] Lang NP, Schild U, Bragger U. Effect of chlorhexidine $(0.12 \%)$ rinses on periodontal tissue healing after tooth extraction. (I). Clinical parameters. J Clin Periodontol. 1994; 21(6): 415-21.

[48] Bragger U, Schild U, Lang NP. Effect of chlorhexidine $(0.12 \%)$ rinses on periodontal tissue healing after tooth extraction. (II). Radiographic parameters. J Clin Periodontol. 1994; 21(6): 422-30.

[49] Saldanha JB, Casati MZ, Neto FH, Sallum EA, Nociti FH, Jr. Smoking may affect the alveolar process dimensions and radiographic bone density in maxillary extraction sites: a prospective study in humans. J Oral Maxillofac Surg. 2006; 64(9): 1359-65.

[50] Vignoletti F, Matesanz P, Rodrigo D, Figuero E, Martin C, Sanz M. Surgical protocols for ridge preservation after tooth extraction. A systematic review.Clin Oral Implants Res. 2012; 23(Suppl5): 22-38. 
